\title{
A new model for the population pharmacokinetics of didanosine in healthy subjects
}

\section{L.S. Velasque ${ }^{1}$, \\ R.C.E. Estrela ${ }^{2}$, \\ G. Suarez-Kurtz ${ }^{2}$ and C.J. Struchiner ${ }^{1}$}

\author{
${ }^{1}$ Programa de Computação Científica, Fundação Oswaldo Cruz, \\ ${ }^{2}$ Coordenação de Pesquisa, Instituto Nacional de Câncer, \\ Rio de Janeiro, RJ, Brasil
}

\author{
Correspondence \\ L.S. Velasque \\ Programa de Computação Científica \\ Fundação Oswaldo Cruz \\ Av. Brasil, 4365 \\ 21045-900 Rio de Janeiro, RJ \\ Brasil \\ Fax: +55-21-2270-5141 \\ E-mail: velasque@fiocruz.br \\ Research supported by $\mathrm{CNPq}$, \\ Fundação Ary Frauzino, and FAPERJ. \\ G. Suarez-Kurtz and C.J. Struchiner \\ are Senior Investigators of CNPq. \\ L.S. Velasque and R.C.E. Estrela \\ were supported by post-graduate \\ fellowships from FAPERJ and \\ Ministério da Saúde, Brazil, \\ respectively.
}

Received June 14, 2005

Accepted March 9, 2006

\begin{abstract}
Didanosine (ddI) is a component of highly active antiretroviral therapy drug combinations, used especially in resource-limited settings and in zidovudine-resistant patients. The population pharmacokinetics of ddI was evaluated in 48 healthy volunteers enrolled in two bioequivalence studies. These data, along with a set of co-variates, were the subject of a nonlinear mixed-effect modeling analysis using the NONMEM program. A two-compartment model with first order absorption (ADVAN3 TRANS3) was fitted to the serum ddI concentration data. Final pharmacokinetic parameters, expressed as functions of the co-variates gender and creatinine clearance $\left(\mathrm{CL}_{\mathrm{CR}}\right)$, were: oral clearance $\left(\mathrm{CL}=55.1+240 \times \mathrm{CL}_{\mathrm{CR}}+16.6 \mathrm{~L} / \mathrm{h}\right.$ for males and $\mathrm{CL}$ $=55.1+240 \times \mathrm{CL}_{\mathrm{CR}}$ for females $)$, central volume $\left(\mathrm{V}_{2}=9.8 \mathrm{~L}\right)$, intercompartmental clearance $(\mathrm{Q}=40.9 \mathrm{~L} / \mathrm{h})$, peripheral volume $\left(\mathrm{V}_{3}=\right.$ $62.7+22.9 \mathrm{~L}$ for males and $\mathrm{V}_{3}=62.7 \mathrm{~L}$ for females), absorption rate constant $\left(K_{\mathrm{a}}=1.51 / \mathrm{h}\right)$, and dissolution time of the tablet $(\mathrm{D}=0.43 \mathrm{~h})$. The intraindividual (residual) variability expressed as coefficient of variation was $13.0 \%$, whereas the interindividual variability of $\mathrm{CL}, \mathrm{Q}$, $\mathrm{V}_{3}, K_{\mathrm{a}}$, and $\mathrm{D}$ was $20.1,75.8,20.6,18.9$, and $38.2 \%$, respectively. The relatively high $(>30 \%)$ interindividual variability for some of these parameters, observed under the controlled experimental settings of bioequivalence trials in healthy volunteers, may result from genetic variability of the processes involved in $\mathrm{ddI}$ absorption and disposition.
\end{abstract}

Key words

- Didanosine

- Pharmacokinetics

- NONMEM

\section{Introduction}

Didanosine (ddI) is a nucleoside analog of adenosine, which prevents replication of human immunodeficiency virus (HIV) by inhibiting HIV reverse transcriptase (1). ddI is a component of highly active antiretroviral therapy drug combinations, used especially in resource-limited settings and among zidovudine-resistant patients $(1,2)$. The pharmacodynamic and pharmacokinetic properties of ddI in HIV-infected individuals and in AIDS patients have been the subject of several reviews (3-6). Regarding the pharmacokinetics of ddI, which is the focus of the present study, important interindividual variability has been reported for several parameters (3-7). Uncontrolled experimental con- 
ditions due to various factors, such as food intake, concomitant administration of other drugs, unbalanced data, etc., could partially explain the variability observed.

Knowledge of the sources and magnitude of this variability can help explain pharmacokinetic differences due to environmental, genetic, and pathophysiologic factors (8). In the present study, we used a broad range of serum ddI concentrations obtained from two bioequivalence trials to develop a new model for this antiretroviral drug in healthy volunteers. Bioequivalence trials are conducted in controlled settings and thus minimize various sources of variability, such as diet, timing of dosing, physical activity, etc. In addition, the pharmacokinetics of highly active antiretroviral therapy components among healthy volunteers may better describe the behavior of these drugs among infected individuals who start therapy early during the course of the infection.

\section{Material and Methods}

\section{Clinical protocol and pharmacokinetic analysis}

The concentrations of ddI in serum samples and the corresponding pharmacokinetic parameters were derived from two bioequivalence studies, in which 48 healthy adult volunteers were enrolled. The studies were conducted in accordance with the revised Decla-

Table 1. Demographics of the study subjects.

\begin{tabular}{lcc}
\hline Characteristic & Value & Range \\
\hline Gender & $24 \mathrm{M} / 24 \mathrm{~F}$ & \\
Age (years) & 26 & $19-48$ \\
Weight $(\mathrm{kg})$ & 64 & $51.7-83$ \\
Height $(\mathrm{cm})$ & 166.5 & $149-187$ \\
Estimated creatinine & 0.14 & $0.10-0.19$ \\
$\quad$ clearance $(\mathrm{mL} / \mathrm{min})$ & & \\
\hline
\end{tabular}

Data are reported as median and range except for gender. Creatinine clearance was calculated by the Cockcroft-Gault equation (14). ration of Helsinki and the rules of Good Clinical Practice (ICH-GCP), the clinical protocols were approved by the Ethics Committee of Instituto Nacional de Câncer, Rio de Janeiro, RJ, Brazil, and all participants provided written informed consent.

Twenty-four volunteers (12 males) were selected for each study according to medical history, physical examination, electrocardiogram, and standard laboratory test results (blood cell count, biochemical profile and urinalysis). These volunteers, whose demographics are shown in Table 1, had not used any investigational drug during the preceding 6 months and prescription drugs other than oral contraceptives were not permitted during the study. In each study, a singledose $(200 \mathrm{mg})$, three-period, six-way, randomized, crossover protocol was used. A detailed description of this experimental design has been published (5). Briefly, in each study, the 24 enrolled volunteers received orally, at weekly intervals, two 100-mg tablets of three different didanosine formulations, one being the reference $\left(\mathrm{Videx}^{\circledR}\right.$, Bristol-Myers-Squibb, Evansville, IN, USA) and the other two generic formulations manufactured in Brazil. The generic formulations used in study No. 1 were manufactured by Laboratório Farmacêutico do Estado de Pernambuco S.A. (LAFEPE, Recife, PE, Brazil) and Indústria Química do Estado de Goiás (IQUEGO, Goiânia, GO, Brazil) whereas in study No. 2 the manufacturers were Instituto Vital Brasil (IVB, Niterói, RJ, Brazil) and Fundação Ezequiel Dias (FUNED, Belo Horizonte, MG, Brazil). Thus, all 48 enrolled volunteers received the reference formulation, whereas each generic formulation was administered to two sets of 24 volunteers. The ddI tablets were administered with $200 \mathrm{~mL}$ water at 7:00 am after an overnight fast, and blood samples $(8 \mathrm{~mL})$ were collected immediately before and 0.25 , $0.5,0.75,1,1.5,2,2.5,3,4,5,6$, and $8 \mathrm{~h}$ post-dosing. The serum was separated within $30 \mathrm{~min}$ and stored at $-20^{\circ} \mathrm{C}$ until the meas- 
urement of ddI concentration using online solid phase extraction coupled to liquid chromatography with positive ion electrospray ionization and tandem mass spectrometry detection (9).

\section{Compartmental pharmacokinetic model}

We used the computer program nonlinear mixed-effect model (NONMEM; double precision, version $\mathrm{V}$, level 1.1), developed by Beal and Sheiner (10). Model fitting was done with NONMEM using the PHOSTOC method (11) and the serum ddI concentrations ( $\mathrm{N}=624$ samples) measured after administration of the reference formulation to the 48 enrolled volunteers. Our choice of an appropriate statistical model was preceded by preliminary modeling exercises when we tried several compartmental models in combination with different types of additive and proportional intraindividual error structures (results not shown). We finally chose the proportional intraindividual error structure and a two-compartment pharmacokinetic model (ADVAN3 TRANS3) using absorption rate constant $\left(K_{\mathrm{a}}\right)$, clearance $(\mathrm{CL})$, volume of the central compartment $\left(\mathrm{V}_{2}\right)$, intercompartmental clearance $(\mathrm{Q})$, volume of the peripheral compartment $\left(\mathrm{V}_{3}\right)$, and duration time of the dose $(\mathrm{D}$, used to model the dissolution time of the tablet: rate $=-2$ ). This latter model provided the best fit among the models tried.

We assumed that interindividual variability of the pharmacokinetic parameters is log-normally distributed, i.e., each parameter value for the $i$ th individual is expressed as $P_{i}=\hat{P}+\exp \left(\eta_{i}\right)$, where $\hat{P}$ is the typical value of the parameter in the population and $\eta_{i}$ is the interindividual error with mean zero and variance $\Omega^{2}$. In addition, intraindividual proportional errors were expressed as $C_{i j}=\hat{\mathrm{C}}_{i j}\left(1+\varepsilon_{i j}\right)$, where $C_{i j}$ is the observed concentration in serum for the $i$ th individual at time $\mathrm{j}, \hat{\mathrm{C}}_{i j}$ is the concentration in serum for the $i$ th individual at time $j$ predicted by the model, and $\varepsilon_{i j}$ is the residual or intraindividual error with mean zero and variance $\sigma^{2}$. Intraindividual and interindividual variabilities were expressed as coefficients of variation (CV). In the model there is only one intraindividual $\mathrm{CV}$ which is calculated as the square root of $\sigma^{2}$. On the other hand, the number of interindividual $\mathrm{CV}$ s depends on the number of random effects included in the model and, therefore, is calculated as the square root of the elements of the matrix $\Omega^{2}$ (11).

\section{Model-building procedure}

We compared an initial set of models that differed from each other by the random effects component associated with each parameter. The model containing random effects associated with all parameters, except $\mathrm{V}_{2}$, minimized the objective function (-2 log likelihood). Using this model as a starting point, we subsequently plotted the individual parameters against co-variates of interest (age, weight, creatinine clearance $\left(\mathrm{CL}_{\mathrm{CR}}\right)$, and gender) to check for trends. The covariates visually identified as possible factors affecting the pharmacokinetic parameters were formally tested using NONMEM. We retained in the model the set of covariates that minimized the AIC statistics (Akaike information criterion $=-2 \log$ likelihood $+2 x$ (number of parameters); 12).

\section{Model validation}

The serum ddI concentrations $(\mathrm{N}=1248$ samples) measured after administration of the four test formulations to the volunteers were compared to the curve predicted by the final model.

\section{Results}

\section{Pharmacokinetic data and bioequivalence assessment}

All the volunteers completed the bioequiva- 
lence trials, and none experienced clinically relevant adverse effects. Figure 1 shows the mean serum concentration-time curves for each ddI formulation, and the pharmacokinetic parameters derived from these curves are presented in Table 2. The values of $\mathrm{C}_{\max }, \mathrm{AUC}_{0-\infty}$, $\mathrm{T}_{\max }, t_{1 / 2}$, or CL/F for the reference formulation did not differ significantly (Mann-Whitney rank sum test) between the two trials, and were in excellent agreement with previous studies in which adult healthy volunteers or HIV-infected individuals were treated with $200 \mathrm{mg}$ oral ddI $(1,13)$. No significant differences in $\mathrm{C}_{\max }, \mathrm{AUC}_{0-\infty}, \mathrm{T}_{\max }, t_{1 / 2}$, or CL/F were observed between the reference and the generic formulations tested in each bioequivalence trial. Bioequivalence assessment indicated that each of the four test formulations fulfilled the adopted bioequivalence criteria (Methods).
Figure 1. Didanosine (ddl) concentrations in the serum of healthy volunteers after single oral doses $(200 \mathrm{mg}$ ) of the drug in two bioequivalence trials. In each trial $(A$, B) 24 volunteers were treated with the reference $\left(V_{i d e x}{ }^{\circledR}\right)$ and two test (generic) ddl formulations (100-mg tablets) at weekly intervals. Data are reported as means $\pm \mathrm{SD}$.

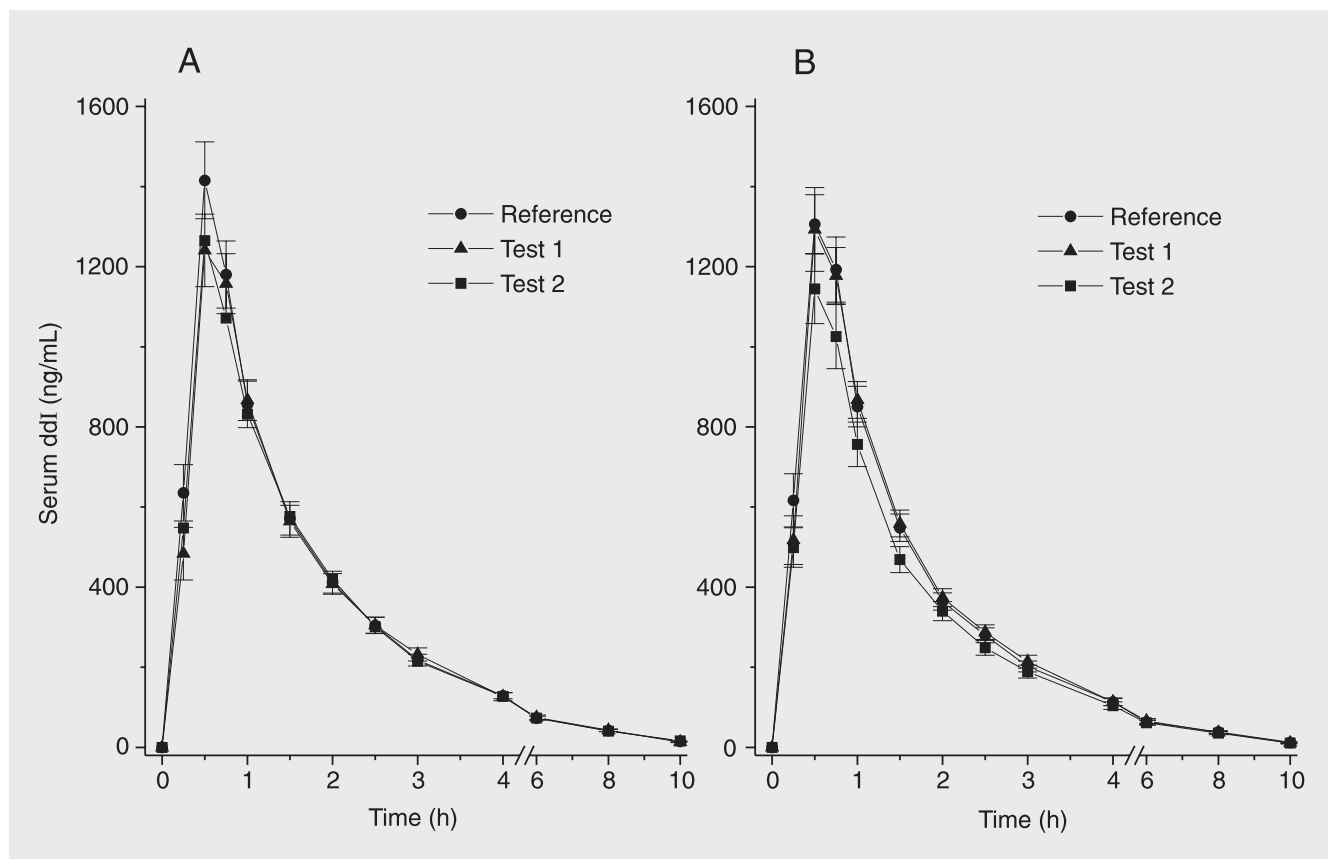

Table 2. Pharmacokinetic parameters of didanosine in healthy volunteers.

\begin{tabular}{|c|c|c|c|c|c|c|c|c|}
\hline \multirow[t]{2}{*}{ Parameter } & \multicolumn{4}{|c|}{ Trial No. 1} & \multicolumn{4}{|c|}{ Trial No. 2} \\
\hline & Reference & Test 1 & Test 2 & $\begin{array}{l}\text { ANOVA } \\
P \text { value }\end{array}$ & Reference & Test 1 & Test 2 & $\begin{array}{l}\text { ANOVA } \\
P \text { value }\end{array}$ \\
\hline$C_{\max }(\mu g / L)$ & $1.5 \pm 0.4$ & $1.3 \pm 0.4$ & $1.3 \pm 0.5$ & 0.60 & $1.4 \pm 0.4$ & $1.4 \pm 0.4$ & $1.2 \pm 0.4$ & 0.21 \\
\hline$A \cup C_{0-\infty}\left(\mu \mathrm{g} \mathrm{h}^{-1} \mathrm{~L}^{-1}\right)$ & $2.2 \pm 0.6$ & $2.2 \pm 0.5$ & $2.2 \pm 0.5$ & 0.86 & $2.1 \pm 0.5$ & $2.1 \pm 0.6$ & $1.9 \pm 0.6$ & 0.23 \\
\hline $\mathrm{T}_{\max }(\mathrm{h})$ & $0.6 \pm 0.1$ & $0.6 \pm 0.2$ & $0.6 \pm 0.2$ & 0.99 & $0.6 \pm 0.2$ & $0.6 \pm 0.2$ & $0.6 \pm 0.1$ & 0.90 \\
\hline$t_{1 / 2}(\mathrm{~h})$ & $1.3 \pm 0.2$ & $1.2 \pm 0.2$ & $1.3 \pm 0.2$ & 0.99 & $1.3 \pm 0.3$ & $1.3 \pm 0.2$ & $1.3 \pm 0.3$ & 0.71 \\
\hline $\mathrm{CL} / \mathrm{F}(\mathrm{L})$ & $184.5 \pm 58.8$ & $173.0 \pm 37.8$ & $190.5 \pm 55.2$ & 0.55 & $187.2 \pm 56.3$ & $183.5 \pm 63.4$ & $224.8 \pm 93.4$ & 0.10 \\
\hline$k_{\mathrm{el}}(\mathrm{h})$ & $0.53 \pm 0.09$ & $0.57 \pm 0.07$ & $0.53 \pm 0.06$ & 0.35 & $0.55 \pm 0.12$ & $0.56 \pm 0.09$ & $0.55 \pm 0.11$ & 0.84 \\
\hline
\end{tabular}

Data are reported as mean \pm SD. Trial No. 1: 24 healthy volunteers ingested $200 \mathrm{mg}$ didanosine as reference (Videx ${ }^{\circledR}$ ) and two test formulations (LAFEPE and IQUEGO) at 7-day intervals. Trial No. 2: 24 healthy volunteers ingested $200 \mathrm{mg}$ didanosine as reference (Videx $\left.{ }^{\circledR}\right)$ and two test formulations (IVB and FUNED) at 7-day intervals. 


\section{Model development}

Parameter estimates for two representative models from the set of models fitted to the data are presented in Table 3. The initial model is best among models that differ only by the presence or absence of random effects associated with the various parameters. The final model is best among models that allow for random effects as well as co-variate effects, i.e., parameter dependence on co-variates. Its objective function is significantly smaller than that of the initial model $(\triangle \mathrm{AIC}$ $=62.45$ ).

$\mathrm{CL}$ is the parameter in the final model most influenced by explanatory variables. Its value varied significantly from the initial to the final model (the 95\% CI's did not overlap). The overlap indicates that the observed difference may not be statistically significant.

Interindividual variability $\left(\omega^{2}\right)$ for several parameters decreased from the starting value in the initial model to the estimated values in the final model. However, the changes were not significant (overlapping $95 \%$ CI's for $\omega^{2}$ ). Interindividual variability estimates, expressed as CV for CL, Q, V $K_{\mathrm{a}}$, and D, were $20.1,75.8,20.7,18.9$, and $38.2 \%$, respectively. Gender had a high and significant (95\% CI's did not include the null value) influence on the parameters CL (16.6 for male) and $\mathrm{V}_{3}$ (22.9 for male). Body weight effects on the various parameters were not significant. The intraindividual variability estimate was $13 \%$ (CV).

Figure 2 shows a plot of the measured versus the model-predicted serum ddI concentrations for the reference formulation in the 48 volunteers studied. The data indicate excellent correlation $(\mathrm{r}=0.98, \mathrm{P}$ value $<0.001$ ). Figure 3 shows typical curves predicted from the model (solid curve) versus the curves observed for healthy volunteers. Analysis of the graph displaying the weighted residuals versus the predicted serum ddI concentrations, for the same model, reveals that
Table 3. Estimates of model parameters for didanosine.

\begin{tabular}{|c|c|c|c|c|}
\hline \multirow{2}{*}{ Parameter } & \multicolumn{2}{|c|}{ Initial model } & \multicolumn{2}{|c|}{ Final model } \\
\hline & Estimate & $95 \% \mathrm{Cl}$ & Estimate & $95 \% \mathrm{Cl}$ \\
\hline$\theta_{\mathrm{CL}(\mathrm{L} / \mathrm{h})}$ & 97.80 & $91.12-104.48$ & 55.10 & $22.5-87.7$ \\
\hline$\theta \mathrm{V}_{2}(\mathrm{~L} / \mathrm{h})$ & 11.00 & $5.58-16.42$ & 9.82 & $3.96-15.68$ \\
\hline$\theta_{Q}(\mathrm{~L} / \mathrm{h})$ & 45.60 & $22.60-68.60$ & 40.90 & $21.08-60.72$ \\
\hline$\theta v_{3}(L / h)$ & 75.40 & $58.28-92.52$ & 62.70 & $50.54-72.46$ \\
\hline$\theta_{K_{\mathrm{a}}(\mathrm{L} / \mathrm{h})}$ & 1.55 & $1.24-1.98$ & 1.51 & $1.15-1.87$ \\
\hline$\theta_{\mathrm{D}}(\mathrm{h})$ & 0.43 & $0.41-0.45$ & 0.44 & $0.40-0.46$ \\
\hline$\theta_{\mathrm{CL}_{\mathrm{CR}}-\mathrm{CL}(\mathrm{L} / \mathrm{h})}$ & - & - & 240.00 & $10-470$ \\
\hline$\theta_{\text {Sex }} V_{3}(L)$ & - & - & 22.90 & $15.76-30.04$ \\
\hline$\theta_{\text {Sex-CL }(L / h)}$ & - & - & 16.6 & $5.33-27.26$ \\
\hline$\omega^{2} \mathrm{CL}$ & 0.06 & $0.02-0.11$ & 0.04 & $0.02-0.05$ \\
\hline$\omega^{2} Q$ & 0.48 & $0.07-0.89$ & 0.57 & $0.07-1.07$ \\
\hline$\omega^{2} v_{3}$ & 0.10 & $0.04-0.15$ & 0.04 & $0.01-0.06$ \\
\hline$\omega^{2} K_{\mathrm{a}}$ & 0.05 & $0.01-0.09$ & 0.04 & $0.00-0.07$ \\
\hline$\omega^{2} D$ & 0.15 & $0.09-0.21$ & 0.15 & $0.09-0.20$ \\
\hline$\sigma^{2}$ & 0.02 & $0.01-0.02$ & 0.02 & $0.01-0.02$ \\
\hline OF & 5150.32 & - & 5081.87 & - \\
\hline AIC & 5172.32 & - & 5109.87 & - \\
\hline
\end{tabular}

$95 \% \mathrm{Cl}=95 \%$ confidence interval; $\mathrm{CL}=$ clearance; $\mathrm{V}_{2}=$ central volume; $\mathrm{Q}=$ intercomportamental clearance; $V_{3}=$ peripheral volume; $K_{\mathrm{a}}=$ absorption rate constant; $\mathrm{D}=$ dissolution time of the tablet; $\mathrm{CL}_{\mathrm{CR}}=$ creatinine clearance; $\mathrm{OF}=$ objective function; AIC (Akaike information criterion) = objective function $+2 x$ (number of parameters).

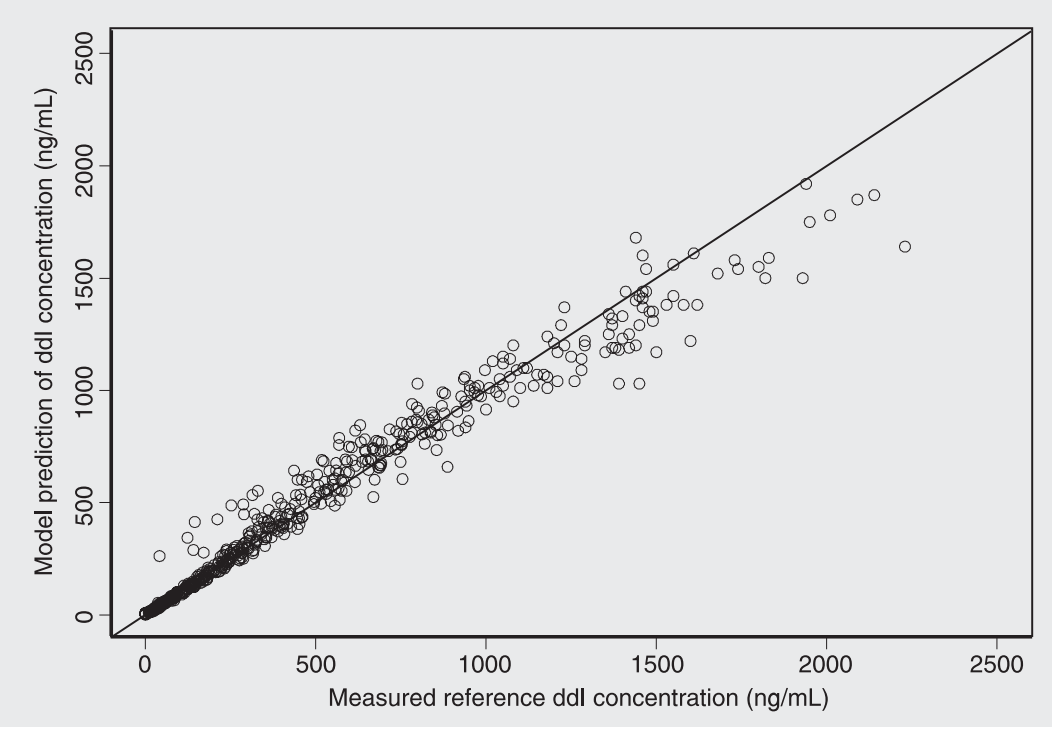

Figure 2. Scatter plot showing the relationship between the serum didanosine (ddl) concentrations measured in 48 healthy volunteers treated with $200 \mathrm{mg}$ of the reference (Videx ${ }^{\circledR}$ ) ddl formulation (abscissa) and the corresponding concentrations predicted by the final model developed in the present study (ordinate). The continuous line is the identity line. 
the residuals are distributed randomly and concentrate within the \pm 2 standard deviation limits (Figure 4).

The final model describing the population pharmacokinetics of ddI is as follows:

Population mean. The set of equations specific for males was $\mathrm{CL}(\mathrm{L} / \mathrm{h})=55.1+240$

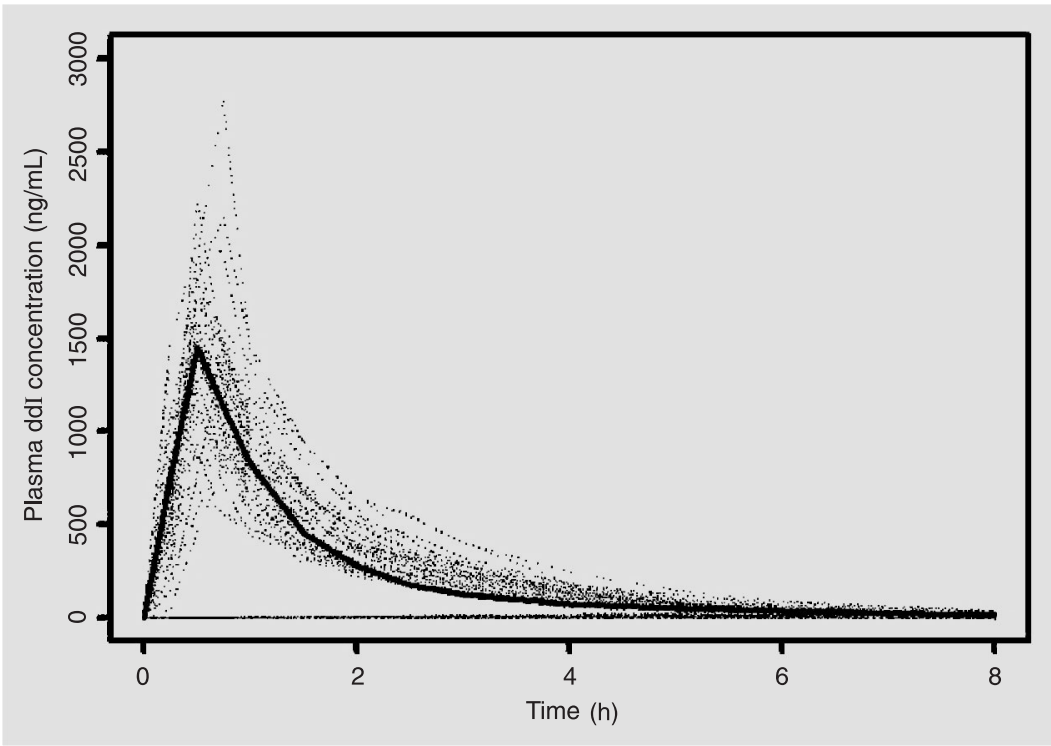

Figure 3. The solid curve is the typical didanosine (ddl) curve predicted by the population model versus time. The dotted lines are plasma ddl concentrations measured in 48 healthy volunteers treated with $200 \mathrm{mg}$ of the reference $\left(V_{i d e x}{ }^{\circledR}\right)$ ddl formulation.

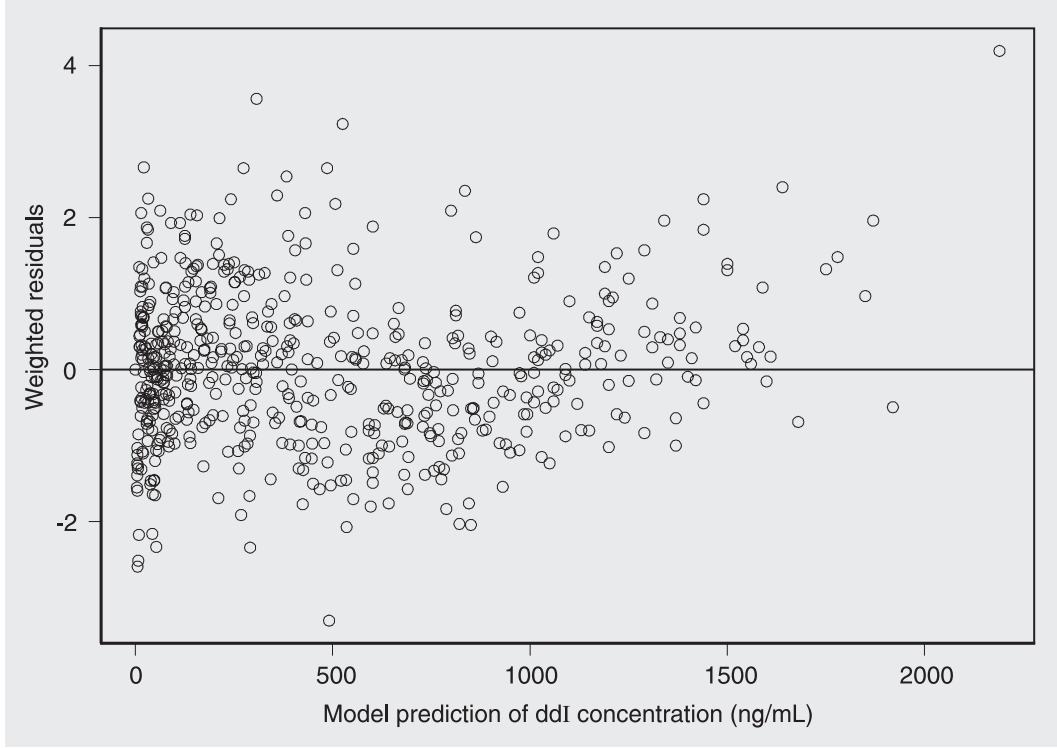

Figure 4. Scatter plot of weighted residual didanosine (ddl) concentration versus the concentration predicted by the final model developed in the present study for reference $\left(\right.$ Videx $\left.{ }^{\circledR}\right)$ formulation. $\mathrm{x} \mathrm{CL}_{\mathrm{CR}}+16.6, \mathrm{~V}_{3}(\mathrm{~L})=62.7+22.9$. Females followed the equations $\mathrm{CL}(\mathrm{L} / \mathrm{h})=55.1+$ $240 \times \mathrm{CL}_{\mathrm{CR}}$ and $\mathrm{V}_{3}(\mathrm{~L})=62.7$.

In addition, the following equations were gender independent: $\mathrm{V}_{2}(\mathrm{~L})=9.82, \mathrm{Q}(\mathrm{L} / \mathrm{h})=$ $40.9, K_{\mathrm{a}}(\mathrm{h})=1.51, \mathrm{D}(\mathrm{h})=0.43$.

Interindividual variability $(\mathrm{CV} \%) . \omega^{2} \mathrm{CL}$ $=20.10 \%, \omega_{Q}^{2}=75.83 \%, \omega^{2} \mathrm{v}_{3}=20.66 \%$, $\omega^{2}{ }_{K_{\mathrm{a}}}=18.95 \%, \omega^{2}{ }_{D}=38.21 \%$.

Intraindividual variability $(\mathrm{CV} \%), \sigma^{2}=$ $12.96 \%$.

\section{Model validation}

The serum ddI concentrations measured after administration of the test formulations to the 48 volunteers were used for validation of the final model. The plot in Figure 5 shows the population curve predicted from the model for both gender (solid curve A and B) versus the curves observed for healthy volunteers who took the test drug.

\section{Discussion}

This is the first study of the population pharmacokinetics of ddI in healthy adults. The software NONMEN and a large number of serum ddI concentrations $(\mathrm{N}=1872)$ from 48 subjects studied under the controlled conditions prevalent in bioequivalence trials were used for model development and validation. Model development was based on data obtained with the reference formulation. The values of the pharmacokinetic parameters $\mathrm{C}_{\max }, \mathrm{AUC}_{0-\infty}, \mathrm{T}_{\max }, t_{1 / 2}$, or $\mathrm{CL} / \mathrm{F}$ for this formulation were in excellent agreement with previous results for healthy adult volunteers or HIV-infected individuals treated with $200 \mathrm{mg}$ oral ddI $(1,13)$. The consistency of these pharmacokinetic data suggests the broader applicability of the population model developed in the present study.

Model diagnostics did not show any deviant pattern. Excellent correlation was observed between the measured and the mo- 
del-predicted serum ddI concentrations for the reference formulation (Figure 2). A distinct feature of our final model was the reduction of the intraindividual (residual) variability to $13 \%$, which implies improved inference properties when compared to previously described models in which the intraindividual variability ranged from 50.2 (6) to $53.9 \%$ (7). The relatively small intraindividual variability in our model can be attributed, at least in part, to the control of potential sources of pharmacokinetic variation in the setting of bioequivalence trials.

The final model included gender as an explanatory variable for $\mathrm{CL}$ and $\mathrm{V}_{3}$. This contrasts with previous studies which excluded effects of gender on the model descriptive parameters for ddI $(6,7)$. One of these studies, however, reported that gender affects the CL of nevirapine (7). Our model identified $\mathrm{CL}_{\mathrm{CR}}$ as another explanatory variable for the CL of ddI, which again contrasts with previous models. At present, we have no explanation for these discrepancies.

The interindividual variability associated with $\mathrm{Q}$ in the final model was highest in the parameter set $(\mathrm{CV}=75.8 \%)$, which points to the intercompartmental clearance of $\mathrm{ddI}$ as the pharmacokinetic process exhibiting the largest heterogeneity among the subjects in the study. Of the other parameters in our final population model, the dissolution time of the tablet - D - was the only one to show substantial $(\mathrm{CV}=38.2 \%)$ interindividual variability. This can be tentatively explained by the different rates of disintegration and dissolution of the ddI tablets in the gastrointestinal tract, despite the $\mathrm{pH}$ buffering included in the formulations. The intraindividual variability of $\mathrm{V}_{3}, \mathrm{CL}$ and $K_{\mathrm{a}}$ was relatively minor ( $\mathrm{CV} \sim 20 \%$ ), but nevertheless removal of each of these terms from the model resulted in significant increases in the objective function and therefore they were retained in the final model.

The final model was validated against the concentration-time observations obtained after administration of the test ddI formulations to the same individuals used for model development. Although distinct formulations were used for model development and validation, we recognize that enrolling different individuals would provide a more stringent validation test, especially in view of the bioequivalence between the reference and each of the test ddI formulations examined.
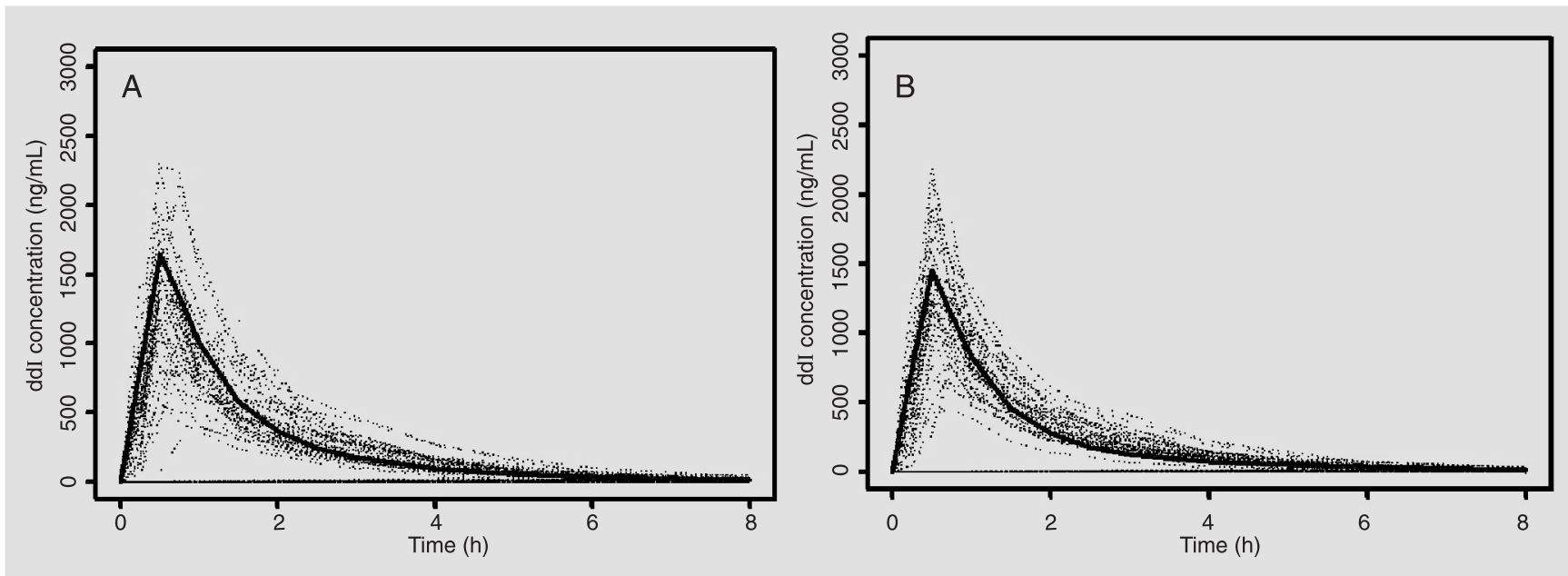

Figure 5. The solid curve is the typical didanosine (ddl) curve predicted by the population model versus time for men and women (A and B, respectively). The dotted lines are the concentrations observed in volunteers who took the test drug. $A$, Model for men: $C L(L / h)=55.1+240 \times C L C R$ $+16.6 ; V_{3}(L)=62.7+22.9 ; V_{2}(L)=9.82 ; \mathrm{Q}(\mathrm{L} / \mathrm{h})=40.9 ; K_{\mathrm{a}}(\mathrm{h})=1.51 ; \mathrm{D}(\mathrm{h})=0.43$. B, Model for women: $\mathrm{CL}(\mathrm{L} / \mathrm{h})=55.1+240 \times \mathrm{CL}_{\mathrm{CR}} ; \mathrm{V}_{3}(\mathrm{~L})=62.7$; $V_{2}(L)=9.82 ; Q(L / h)=40.9 ; K_{\mathrm{a}}(\mathrm{h})=1.51 ; \mathrm{D}(\mathrm{h})=0.43$. 
We report the development and validation of a population pharmacokinetic model for the antiretroviral drug, ddI, using data from two bioequivalence studies in which generic formulations manufactured in Brazil proved to be bioequivalent with the reference ddI tablet formulation available internationally. The final model achieved lower intraindividual (residual) variability than previous models reported in the literature, which were developed using data from HIVinfected individuals $(6,7)$. Because our model was based on ddI data from healthy volunteers, it might be more relevant to the pharmacokinetics of ddI administered to HIV-infected patients early in the course of the infection. We observed that the interindividual variability in the model descriptive parameters remained important even in the controlled setting of bioequivalence trials. Our observation that the interindividual variability in the model descriptive parameters remained important even in the controlled setting of bioequivalence trials may suggest that pharmacogenomic factors play a relevant role in the disposition of ddI in humans.

\section{References}

1. Perry CM, Noble S. Didanosine: an updated review of its use in HIV infection. Drugs 1999; 58: 1099-1135.

2. Faulds $\mathrm{D}$, Brogden RN. Didanosine. A review of its antiviral activity, pharmacokinetic properties and therapeutic potential in human immunodeficiency virus infection. Drugs 1992; 44: 94-116.

3. Adams JM, Shelton MJ, Hewitt RG, Grasela TH, DeRemer M, Morse GD. Relationship between didanosine exposure and surrogate marker response in human immunodeficiency virus-infected outpatients. Antimicrob Agents Chemother 1998; 42: 821-826.

4. Harb GE, Mandema JW, Delahunty T, Benowitz NB, Coleman R, Sheiner LB, et al. Population pharmacokinetics of didanosine in patients with human immunodeficiency virus infection. $J$ Infect Dis 1996; 173: 273.

5. Morse GD, Shelton MJ, Ho M, Bartos L, DeRemer M, Ragni M. Pharmacokinetics of zidovudine and didanosine during combination therapy. Antiviral Res 1995; 27: 419-424.

6. Pai SM, Shukla UA, Grasela TH, Knupp CA, Dolin R, Valentine FT, et al. Population pharmacokinetic analysis of didanosine (2',3'dideoxyinosine) plasma concentrations obtained in phase I clinical trials in patients with AIDS or AIDS-related complex. J Clin Pharmacol 1992; 32: 242-247.

7. Zhou XJ, Sheiner LB, D'Aquila RT, Hughes MD, Hirsch MS, Fischl MA, et al. Population pharmacokinetics of nevirapine, zidovudine, and didanosine in human immunodeficiency virus-infected patients. The National Institute of Allergy and Infectious Diseases AIDS Clinical Trials Group Protocol 241 Investigators. Antimicrob Agents
Chemother 1999; 43: 121-128.

8. Kalow W. Interethnic differences in drug response. In: Kalow W, Meyer UA, Tyndale R (Editors), Pharmacogenomics (Drugs and the Pharmaceutical Sciences: A Series of Textbooks and Monographs). New York: Marcel Dekker Inc.; 2001. p 109-134.

9. Estrela RC, Salvadori MC, Raices RS, Suarez-Kurtz G. Determination of didanosine in human serum by on-line solid-phase extraction coupled to high-performance liquid chromatography with electrospray ionization tandem mass spectrometric detection: application to a bioequivalence study. J Mass Spectrom 2003; 38: 378-385.

10. Beal SL, Sheiner LB. NONMEM User's Guide. NONMEM Project Group. San Francisco: University of California at San Francisco; 1992.

11. Boeckmann AJ, Sheiner LB, Beal SL. NONMEM User's Guide - Part V. NONMEM Project Group. San Francisco: University of California at San Francisco; 1994.

12. Burhnam KP, Anderson DR. Model selection and multimodel inference - A pratical information-theoric approach. New York: SpringerVerlag; 2002.

13. Burger D, Meenhorst P, Mulder J, Henrichs J, Frissen J, Kroon F, et al. Substitution of didanosine sachets by chewable tablets: a pharmacokinetic study in patients with AIDS. J Acquir Immune Defic Syndr Hum Retrovirol 1995; 10: 163-168.

14. Cockcroft DW, Gault MH. Prediction of creatinine clearance from serum creatinine. Nephron 1976; 16: 31-41. 